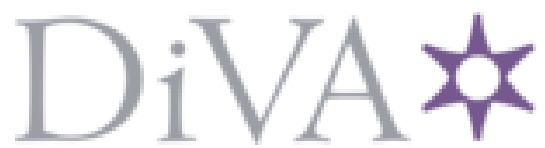

http://www.diva-portal.org

Postprint

This is the accepted version of a paper presented at 2O17 IEEE 21st International Enterprise Distributed Object Computing Conference Workshops and Demonstrations (EDOCW 2017).

Citation for the original published paper:

Hacks, S., Brosius, M., Aier, S. (2017)

A Case Study of Stakeholder Concerns on EAM

In: (pp. 50-56).

https://doi.org/10.1109/EDOCW.2017.17

N.B. When citing this work, cite the original published paper.

Permanent link to this version:

http://urn.kb.se/resolve?urn=urn:nbn:se:kth:diva-255637 


\section{A Case Study of Stakeholder Concerns on EAM}

\author{
Simon Hacks \\ Research Group Software Construction \\ RWTH Aachen University \\ Aachen, Germany \\ simon.hacks@swc.rwth-aachen.de
}

\author{
Maximilian Brosius, Stephan Aier \\ Institute of Information Management \\ University of St. Gallen \\ St. Gallen, Switzerland \\ \{maximilian.brosius, stephan.aier\}@unisg.ch
}

\begin{abstract}
As a result of growing complexities in business processes, information systems, and the technical infrastructure, a key challenge for enterprise architecture management (EAM) is to guide stakeholders from different hierarchical levels with heterogeneous concerns. EA deliverables, such as models or frameworks, are often highly comprehensive and standardized. However, these can hardly be applied without greater adaption. Although the literature selectively covers approaches for tailoring EA deliverables closer to the concerns of affected stakeholders, these approaches are often vague or not very differentiated. In the paper at hand, we aim at introducing a stakeholder perspective to EAM research that considers stakeholder concerns on EAM across hierarchical levels. To this end, we conduct a case study: Our results show homogenous concerns among stakeholders on EA deliverables. In turn, we found different concerns on the role of EAM in applying these deliverables, dependent on the hierarchical level of stakeholders. These findings stress the necessity for a more differentiated understanding of stakeholder concerns on EAM. Finally, we discuss the implications of our findings for an exemplary EAM approach.
\end{abstract}

Keywords-enterprise architecture management (EAM); stakeholder concerns; hierarchical level; case study

\section{INTRODUCTION}

An increasing number of information system (IS) change and development projects focus the realization of technical solutions for local business needs. Enterprise architecture (EA) is a prominent discipline that aims at guiding local IS endeavors through a holistic view on the fundamental structures, design, and evolution principles of the overall organization [1]. By applying this holistic view, IS projects become more aligned with enterprise-wide objectives, which leads to reduced complexities as well as integration efforts in the overall corporate IS landscape.

Due to the growing number of IS change and development projects, however, EA management (EAM) is confronted not only with the challenge of aligning IS, but also with the challenge of responding to different stakeholders and their respective concerns ${ }^{1}$. More importantly, EAM has to respond to stakeholders concerns from different levels of hierarchy and positions of authority command, ranging from local IS developers or department leaders to senior business and IT executives [3].

Standardized architecture models and frameworks, such as The Open Group Architecture Framework (TOGAF), entail valuable EA deliverables. However, these can hardly be applied without greater adaption. A major reason lies in the high degree of standardization and comprehensiveness, by which EA deliverables are aimed to become applied to a wide range of stakeholders and use cases. Yet, leaving EA deliverables untailored to the concerns of stakeholders jeopardizes guidance effects on both the IT and business side [4].

A few publications cope with the question of how to tailor EA deliverables to different stakeholder concerns (e.g., [4-7]). Despite their discussion around EA deliverables, the extant literature still lacks a mere concrete intuition to stakeholder concerns on EAM. More specifically, there is a research gap about the hierarchical differences of stakeholder concerns and their implications to EAM. Recognizing these shortcomings in the extant literature, we formulate our research question (RQ):

\section{$R Q:$ What are the hierarchical differences of stakeholder} concerns on EAM?

In order to identify stakeholder concerns on EAM along hierarchical differences, we opt for a case study to investigate our research objective in a real-life context. Our findings demonstrate different concerns on EA deliverables and EAM, depending on the hierarchical level of the respective interviewee. Using TOGAF exemplarily as standardized and highly comprehensive EAM approach, we discuss the implications of our findings for future research.

This paper is structured as follows: Firstly, we review related work on stakeholder perspectives in the EA literature and outline the research gap. Secondly, we present the research method, in which we provide the case description, data collection, and scheme-guided classification. Along the developed classification scheme, we present our analysis of stakeholder concerns, reflecting EA deliverables and EAM. Finally, we discuss the implications of our analysis.

\footnotetext{
${ }^{1}$ We define concerns as relevant interests that pertain to system development, its operation or other important aspects to stakeholders [2].
} 


\section{RELATED WORK}

The term "stakeholder" refers to a "group or individual who can affect or is affected by the achievement of the organization's objectives" [8]. Stakeholders are identified by their legitimate interests in the organization. However, these interests are often self-serving and hence impose a concern for the organization to manage [9].

Notably, stakeholders are not randomly important [10]. Rather, the identification of relevant stakeholder concerns for a specific purpose becomes the first and most essential priority to any management endeavor $[11,12]$. This necessity becomes even more decisive in the context of enterprise-wide IS initiatives - such as EAM - that highlight various interrelated stakeholders with competing, depending, and often conflicting concerns [13].

EA stakeholders are typically representatives from the IT side (e.g., system developers) and the business side (i.e., end users who make use of the developed systems) [3]. These have different concerns regarding the development of systems, which are manifested in relation to other stakeholders' goals, expectations, requirements, dependencies, and risks [2]. EA stakeholders span different hierarchical levels: Typically, corporate functions, such as senior executives (e.g., CIO), department heads, projects leads as well as local business and IT affiliates, among others, are considered [3].

\section{A. Stakeholder Concerns in EAM research}

In order to approach the diversity of stakeholder concerns, the more prominently applied EA deliverables (e.g., frameworks, models) are dedicated to a high degree of comprehensiveness and standardization. The Open Group Architecture Framework [3], for example, describes, on over 600 pages, means and methods for designing, planning, implementing, and governing the EA. Notwithstanding its adherence to a rather high level of comprehensiveness, what TOGAF and other EA frameworks have in common is that they simultaneously promote the necessity to tailor the EA to different groups of stakeholders and situational specificities [3].

To date, only a few publications deal with the question of how to tailor EA deliverables to the concerns of different stakeholders. For instance, Lindström et al. [5] compared different architecture frameworks to the concerns of CIOs. They found that two important stakeholder concerns (i.e., quality of interplay between business and IT, business cost reduction) were not met by the architecture frameworks, concluding that EA frameworks and models should allow greater modifications and inclusiveness of information.

Van der Raadt et al. [14] focused EA from two different stakeholder perspectives, presenting their perceptions in a cognitive map. Their findings promoted proper mutual understanding and higher degrees of collaboration between architects and EA stakeholders. Moreover, mutual understanding and collaboration were found to have a positive effect on EA service delivery as well as on stakeholders' willingness to actively participate in the EA function.
Kurpjuweit and Winter [4] focused on how to design the modeling and analysis of EA in a stakeholder-oriented way. They assumed stakeholders' diversity to facilitate EA models and analyses, gaining insights from dependencies and interrelations in the EA. To this end, they developed the design of an integrated, navigable, and expandable view-point system for EA modelling and analysis.

Buckl et al. [15] applied a pattern-based perspective to EAM. Patterns refer to proven solutions on recurring problems, such as in methodological, viewpoint, or information model aspects. On the example of the architecture development method (ADM) in TOGAF, they discussed how generic guidelines can be complemented via selected EAM patterns. Their findings showed that EAM patterns were especially useful for guiding highly prioritized EA concerns with corresponding viewpoints and information models.

Aleatrati Khosroshahi et al. [16] published the latest version of the Enterprise Architecture Management Pattern Cata$\log$, a collection of stakeholder concerns, methodology-, viewpoint- and information model patterns to implement EAM in organizations. At the core of their work is the identification of EAM concerns among diverse business (e.g., business analyst, project manager, head of department) and IT-affected stakeholders (e.g., software developer, IT project controller, CIO). Concerns on EAM, among others, comprised the communicated added value of EAM, detection of consolidation potentials, the removal of monolithic applications, and the measurement of changes in the application landscape. The authors addressed each of these concerns by one or more methodology patterns.

\section{B. Research Gap}

EA literature reveals a strong correlation between meeting stakeholder concerns and the necessity of adapting EA deliverables [15]. While EA deliverables must be comprehensive and standardized to become applicable to different stakeholder groups and potential use cases, yet these deliverables often do not provide a more concrete implication on how to adapt them to specific concerns [3]. In turn, EA literature has promoted several approaches for deliverable adaption [4]. However, the proposed solutions mainly focus conceptual [6] and not management-related aspects of EA deliverables.

EAM literature has emphasized architectural guidance in a deliverable-centered fashion, promoting the use of rules and plans from a centralized position in the hierarchy of an organization $[15,17]$. Despite the body of literature studying the adaption of EA deliverables, stakeholder concerns on EAM remain largely undiscussed. Specifically, with regards to hierarchical differences of stakeholder concerns, extant research lacks mere explicit intuitions on EAM.

Recognizing the necessity of EAM in realizing its guidance through EA deliverables, we argue the deliverable-centered discourse in the extant literature [4] as one possible solution for tackling different stakeholder concerns. However, this may not be sufficient for fully coping with hierarchical differences among stakeholders. For this reason, we stress the need for a more hierarchy-facilitated perspective on stakeholder concerns. To the best of our knowledge, there is no comparable study in dealing with the exposed research gap yet. 


\section{CASE DESCRIPTION}

The case organization is one of the leading insurance providers in the German-speaking market. About 30,000 employees and 16,000 associated agents count toward the workforce of the company. The organization gains revenues of over 16 billion Euro and manages investments of 135 billion Euro. Furthermore, the case organization has several subsidiaries: One of them is the internal IT service provider, in which we conducted our case.

The IT service provider employs around 1,400 employees. These are responsible for operations and development of technological solutions for the whole organization, including all of its subsidiaries. The IT provider began establishing EAM initiatives in 2008 and currently hosts two EAM units: The first unit, "architecture management", employs twelve members, being responsible for application development. The second unit, "infrastructure architecture management", hosts fifteen internal and thirty external (e.g., consultants) members, who are responsible for infrastructure management (e.g., operations of servers). As regulatory instances, both units are responsible for all EA-related questions, ranging from EA development to EA implementation and EA maintenance.

\section{RESEARCH METHOD}

The research method follows a two-step process, starting with the data collection, followed by a scheme-guided classification for presenting and discussing the data.

\section{A. Data Collection}

A single case fits our purpose of gaining a first, in-depth reflection of hierarchical differences of different stakeholder concerns in a real life scenario [18]. A case study is further suitable to shed light on the phenomenon of interest from different perspectives [18], which fitted our research objective of tackling stakeholders from different hierarchical levels with diverse concerns (Figure 1). Following Patton [19], we decided to conduct our case study by a series of open-ended interviews, using a fixed set of questions for all interviewees. Choosing this method over a fixed set of questions thereby contributed to a high degree of comparability of our respondents' answers.

In line with our research objectives, interview questions were focused on EA deliverables as well as stakeholder con-

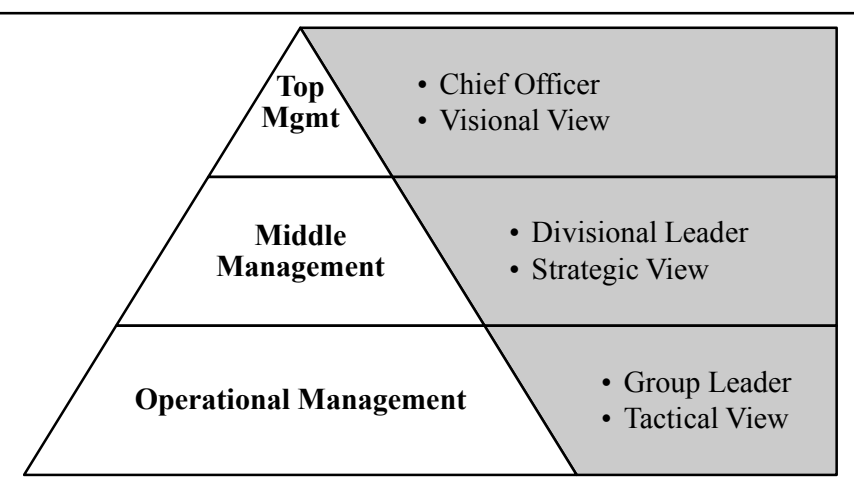

Fig. 1. EAM's Different Groups of Stakeholder

cerns on EAM. In total, 36 questions were developed. In order to guide our participants structured through the interviews, the developed questions were assigned into seven sequential blocks of questions (i.e. introduction, perception, points of contact, strategic role, architectures, policies, and solution architectures).

The interviews lasted up to 60 minutes. In total, 38 stakeholders participated in the interviews. The chosen stakeholders represented different hierarchical levels (see Figure 1): Operational management (e.g., group leaders, solution architects), middle management (e.g., division leaders, project leads), and top management (i.e. management board). The operational management was represented by 18 interviewees, the middle management by 15 interviewees, and the top management by five interviewees.

All interviews were recorded and transcribed. Finally, the transcripts were synthesized into a comprehensive summary of 1,042 stakeholder concerns for further classification (see scheme-guided classification).

\section{B. Scheme-guided classification}

In order to synthesize and present the collected 1,042 stakeholder concerns, we used a scheme-guided classification. Following the two steps by Miles and Huberman [20] as well as Eisenhardt [21], two of the three involved researchers accounted responsible for the early analysis and coding.

TABLE I. STAKEHOLDER CONCERNS ON EAM

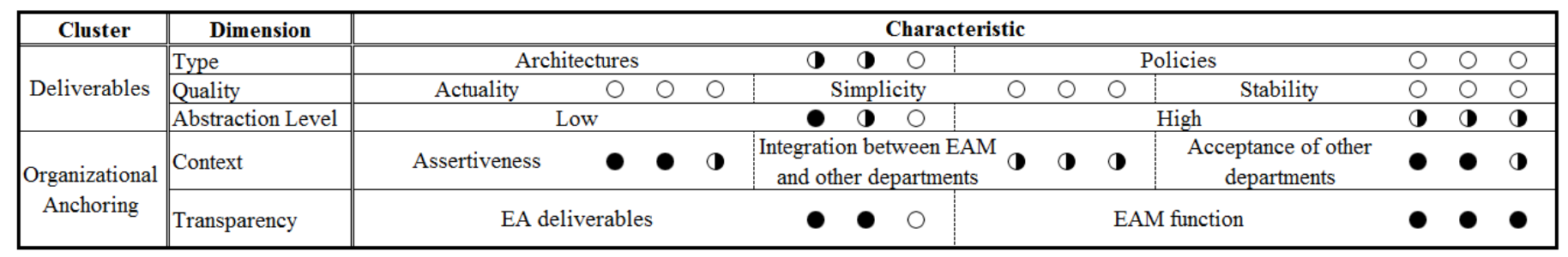

$$
\begin{aligned}
& \text { - rarely mentioned } \\
& \text { - frequently mentioned } \\
& \text { - often mentioned }
\end{aligned}
$$

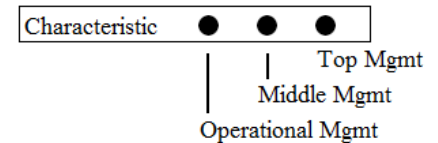


Early analysis. At the outset, the consolidated transcript was screened, encompassing 1,042 synthesized statements of stakeholder concerns. These statements were studied for the identification of common characteristics: Statements referring to the same concern - or to similar characteristics of one and the same concern - were assigned to one dimension. Finally, the early analysis resulted in five dimensions of stakeholder concerns (Table I). Consistent with our research objectives, these two dimensions differentiate EA deliverables and organizational anchoring.

Coding. Counting toward the most comprehensive and widest implemented EAM approaches [3], we applied TOGAF for developing the terminology of dimensions and for facilitating these dimensions with illustrative characteristics (Table I). The first dimension of stakeholder concerns gave particular rise to the differences among EA deliverables: Type [3]. Type refers to EAM artifacts, being contractually specified and formally reviewed. In line with TOGAF, we differentiated two main groups of deliverables that interviewees reported, namely, architectures (e.g., as-is architectures, business domain model, infrastructure blueprint) and policies (e.g., principles, documentation rules, decision boards, standards).

The second dimension introduces quality to EA deliverables. Following TOGAF [3], three quality criteria were added to consolidate the respondents' concerns: Actuality, stability, and simplicity. While EAM needs to deliver in quality to a wide range of diverse stakeholders, deliverables are required to maintain a certain degree of abstraction [3], which represents the third dimension of the classification scheme. More general, we differentiate high and low levels of abstraction. The fourth dimensions, context, describes the environmental setting and specificities, in which EAM operates [3]. Context spans the set of expectations toward and acceptances of the EAM function, which prevail in the organizational environment, as well as the structural arrangement of organizational units. This includes, among others, the sufficient staffing of architecture relevant tasks as well as an organizational culture centered on architectural policies. Finally, transparency accounts as fifth dimension of the classification scheme [3], focusing on "the perceived quality of intentionally shared information" in the context of architectural guidance [22]. Transparency applies to EA deliverables as well as the organizational anchoring of the EAM function.

Classification scheme. Our final classifications scheme is comprised of five major dimensions and twelve facilitating characteristics (Figure 1).

\section{STAKEHOLDER CONCERNS}

In the following, we present the collected stakeholder concerns along the dimensions of the classification scheme. All concerns were classified by the percentage of stakeholders of each group reporting to them: Rarely (less than 33\% of stakeholders), frequently (between $33 \%$ and $66 \%$ ), and often (more than $66 \%$ ).

\section{A. Type}

Architectures. Interviewees from the operational and middle management level were similarly concerned with architec- tures (see Table I). The most often reported deliverable was the as-is architecture. Thereby, concerns referred to infrastructure components on the technology layer as well as the application layer, i.e. what applications exist and how they are connected.

Members of the top management were less concerned than members of the operational and middle management. As a result of different use cases, interviewees of the top management mentioned as-is architectures solely as a means of steering.

Policies. Policy concerns, discussed mainly in the context of project management and reporting activities, referred to complexity-related (e.g., "documents should be formulated in a simpler way.") and transparency-related (e.g., "the added value and the function of a policy should be clear.") aspects. A further concern, according to the interviewees, referred to guidelines (e.g., architecture principles): On the one hand, interviewees acknowledged the usefulness of architecture principles. On the other hand, usefulness of architecture principles appeared to be of limited value once generating too much effort for following them (e.g., "developers ignore the architecture principles if it is inconvenient."). Moreover, interviewees concerned missing assertiveness of the EAM, thus, developers are not forced to comply with policies (e.g., "violation of the architecture principles will not be sanctioned.").

\section{B. Quality}

Actuality. A rarely mentioned concern among all hierarchical levels of stakeholders was actuality. Interviewees described actuality as expectancy for the case of using the deliverables of EAM (e.g., "... should be up-to-date"). Particularly, actuality has been emphasized for application layer (e.g., detailed information about applications, communication between applications) due to its frequency of usage as a basis for architecture design decisions (e.g., "the provided as-is application layer is not up-to-date. This is insufficient if we were about to use it.").

Simplicity. Another mentioned characteristic of quality was simplicity. Participants stressed architecture principles to function as guidelines for application developers: Firstly, there should be a limited number of principles to enable affected persons to keep the overview. Secondly, principles should not be too complex (e.g., "a multi pages document comprises all information about the principle privacy and security."). Finally, deliverables should be easily accessible (e.g., simple search functionalities along all deliverables, no spread of information in different sources).

Stability. Interviewees expected stability in the management of deliverables (i.e., design, maintenance, retirement). Stakeholders throughout all hierarchical levels differentiated two facets of this concern: First, names of individual deliverables had to be changed only on purpose. Second, policies should not be changed too often and not too fundamentally.

More generally, quality concerns were reported as important to avoid confusion (e.g., "if a certain product of EAM is renamed, it will take some time to recognize whether the product is renamed or completely retired.") and to reduce unintentional effort (e.g., "changing policies leads to additional effort, because coaching is needed to internalize the changes."). 


\section{Abstraction Level}

Low. As shown in Table I, concerns for a low abstraction were mentioned very divergently by the stakeholder levels. Apparently, this characteristic was most relevant for operational management stakeholders. These stakeholders were particularly concerned with concrete instructions and for operations usable information. In detail, coaching in projects was requested in order to learn how to build applications in line with existing policies. Additionally, detailed information on applications as well as specific information on business structures was concerned. Compared to operational management stakeholders, members from the middle and top management valued a low abstraction level less high.

High. The need for a high abstraction level was reported throughout all stakeholder levels similarly (see Table I). Concerns were related to the degree of details among steering decisions, which commonly necessitated a higher level of abstraction. Moreover, a high level of abstraction also favored better visualization of relevant information to be presented.

\section{Context}

Assertiveness. As Table I illustrates, assertiveness played an important role for all interviewees. Concerns of assertiveness dealt with EAM as a control and enforcement function in implementation processes. This control and enforcement function was particularly highlighted by the interviewees due to missing sanctions on non-architecture-compliant technological developments and non-principle conform behavior.

Integration between EAM and other departments. Like assertiveness, the integration of EAM was stated as important from all stakeholder levels (see Table I). Concerns referred to the necessity to integrate neighboring departments into the development of EA policies. Especially, the involvement of the strategy department was mentioned. Moreover, the representatives of business concerned further involvement in planning processes of EA business aspects (e.g., modelling business functions and assigning those to applications).

Acceptance of other departments. Interviewees argued that IT departments do not follow the architecture policies for different reasons. Some are not aware of the guidelines. Others are aware, however, do not follow policies or do not have the resources to follow. On the one hand, interviewees stated the need for budget to cope with the additional efforts that are generated by the implementation of architectural principles. On the other hand, budget issues concerned the sufficient staffing of the EAM function.

\section{E. Transparency}

EA deliverables. EA deliverables were often mentioned by interviewees from the operational and middle management. In contrast, interviewees from the top management quoted deliverables occasionally (see Table I). Concerns referring to transparency mainly resulted as a lack of knowing EA deliverables (e.g., "I did not know that there exists such a thing like an application portfolio."). Consequently, interviewees suggested a clearer communication of the existing deliverables to the stakeholders.
EAM function. The EAM function is often acknowledged across all stakeholder groups (Table I). The interviewees often wondered about the process of generating EA deliverables. Most importantly, the question of how decisions regarding architectures are taken remains unclear (e.g., "what are the decision processes?"; "what are the inputs of the deliverables?"). Interviewees suggested a higher degree of transparency of the EAM processes as well as their communication toward affected stakeholders.

\section{DISCUSSION}

The review of stakeholder concerns brings about two major distinctions: We found relatively homogenous concerns among stakeholders on EA deliverables, such as type, quality, and abstraction level. In turn, heterogeneous concerns were found on the role of EAM (i.e. context, transparency), depending on the hierarchical level of the interviewees. In the following, we discuss these two distinctions of responses along each of our five framework dimensions. For purpose of demonstrating the implications to EAM approaches, we use TOGAF as illustrative example.

\section{A. Type}

TOGAF [3] differentiates architectures along three levels of granularity: Strategic, segment, and capability. The stakeholders of these levels - as entailed by TOGAF - correspond to the identified stakeholder groups in section IV. Consequently, TOGAF states the concern to deliver in different granularity levels of architecture deliverables to different stakeholder. Unlike TOGAF, interviewees of the executive management hardly reported concerns on architectures, which indicate less need for such a granularity level.

Policies are used to ensure implementation governance of the architecture [3] as they set the frame to steer the application development and to describe the architecture compliance review process. Furthermore, TOGAF lacks a separation between different stakeholder groups, too. This lack of differentiation is in line with our case results, finding no differences with regards to the hierarchical level of stakeholders (such as on policies).

\section{B. Quality}

In TOGAF, actuality does not apply to all EA deliverables [3], which is primarily due to the high level of abstraction. In contrast, our case results promoted actuality with the same importance among all hierarchical levels of stakeholders.

Understandability is one of the quality concerns TOGAF defines for EA deliverables [3]. However, interviewees did not acknowledge the term understandability, but the term simplicity. Understandability selectively reflects simplicity. Interviewees brought up simplicity, though, in context of all deliverables. This is in contrast to the use of understandability in TOGAF. Further, stakeholders concerned not only an understandable design of deliverables, but also their easy access. This is also reflected in simplicity, but not in understandability.

TOGAF defines stability as one of the quality criteria [3]. However, it does not differentiate a relevancy between differ- 
ent stakeholder groups. Within our interviews, we were not able to confirm such a differentiation either.

In general, the concern for quality characteristics was low among all stakeholder levels. This may stem from the lack of knowledge regarding EA deliverables among our interview participants.

\section{Abstraction Level}

TOGAF emphasizes the development of EA deliverables in a stakeholder-specific fashion [3]. While promoting a stakeholder focus, TOGAF does not entail a method to systematically identify stakeholders and their concerns. Moreover, TOGAF proposes a low level abstraction of architectures for operational managers [3]. In line with TOGAF, our interviewees concerned deliverables with low abstraction, too. As opposed to TOGAF, stakeholders who belong to the middle management concerned also deliverables with a low abstraction level.

Compared to low abstraction levels of EA deliverables, stakeholders also raised the need for high levels of abstraction. Particularly, members of the operational management concerned the need for a high abstraction of EA deliverables, yet also stated a high abstraction level to be more feasible for middle as well as top management.

\section{Context}

Interviewees often brought up assertiveness to concern controlling the implementation, enforcing policies, and committing adherence to authority structures established by the EAM. Certainly, only the last aspect is reflected properly in TOGAF by its term discipline. TOGAF defines discipline as "a commitment to adhere to procedures, processes, and authority structures established by the organization" [3]. Therefore, we take over interviewees' assertiveness to reflect all pointed out aspects.

Interviewees across operational and middle management named assertiveness of the EAM as an important characteristic. Only stakeholders of the top management were less concerned with assertiveness. Interviewees assumed that this may stem from the fact that assertiveness regarding policies is most important on non-strategic levels. Thus, on non-strategical levels, deliverables are produced which must comply with policies.

According to TOGAF [3], cross integration is an important success factor of architecture governance which is part of the organizational context. Similarly, interviewees stated the necessity to integrate neighboring departments into the development of EA policies. Contrary to TOGAF, our case analysis did not bring about any separation between the stakeholder groups with regards to the characteristic integration.

Acceptance is selectively reflected in TOGAF [3] as one of the cornerstones for realizing conformity to procedures, processes, and authority structures. Surprisingly, we observed only partial interest among interviewees of the top management. This correlates with the promoted need for a better staffing of the operational management, steered by the top management.

\section{E. Transparency}

According to TOGAF, transparency is the availability of all implemented actions and their decision support for authorized organizations and provider parties [3]. Moreover, TOGAF promotes the necessity of transparency also for understanding deliverables [3]. One facet of transparency mentioned by our interviewees dealt with the communication of existing $E A$ deliverables. On the one hand, particular members of the operational and middle management, who are often guided by EA deliverables (e.g., complying with policies), inherently promoted transparency. On the other hand, members of the top management who are not guided by EA deliverables were less concerned by transparency of deliverables. In contrast, we could not find any separation for transparency regarding the $E A M$ function.

\section{F. Implications}

Discussing our findings on the illustrative example of TOGAF, we conclude four implications for EAM approaches in general. Firstly, two rather than three different levels of abstraction for EA deliverables appear to be sufficient: On the one hand, interviewees stated only concerns regarding two different layers, namely low and high level. On the other hand, stakeholders of the top management throughout our interviews appeared not to be interested in architectural deliverables.

Secondly, some concerns are not entirely reflected in TOGAF. For example, the definition of quality concerns should be expanded to consider all EA's deliverables, not being limited to architecture principles. While interviewees were concerned with simplicity, simplicity is not referred to in TOGAF's terminology. The term understandability selectively reflects simplicity, but not in a fully comprehensive manor. Therefore, it may be helpful for TOGAF's completeness to either replace understandability with simplicity or to add simplicity to deliverable qualities.

Thirdly, we identified a transparency concern for the EA function and its deliverables. Future research should elaborate on strategies how EAM departments could more effectively advertise their deliverables and the EA function itself.

Lastly, our results confirm TOGAF's standardization ambitions. However, our results also show the need for a stakeholder specific tailoring, following stakeholders who expect different abstraction levels of deliverables according to their hierarchical position in the organization.

Apart from the improvement potentials of TOGAF, our investigation delivered additional implications for EAM research: Interviewees were just modestly interested in quality criteria of EA deliverables. Future research can elaborate on this in organizations where EA deliverables are better known. Moreover, the results may be transferable to other domains in organizations which have crossing functions, like IT-security or strategy, which can be evaluated in the future.

\section{CONCLUSION}

Our study contributes to the existing literature on stakeholder concerns by introducing a differentiation of hierarchical stakeholder levels. In the study at hand, we focused stakeholder 
groups from the operational management, middle management, and top management. For most concerns, the needs of the different stakeholder groups were found to be rather homogenous. However, concerns on architecture, abstraction level, assertiveness, acceptance of other departments, and transparency of EA deliverables were discovered to be rather heterogeneous.

This research has some limitations. Firstly, all interviewees belonged to the IT side. Consequently, the results may be limitedly applicable to the business side. As one of the main objectives of EAM is to incorporate the business side, future research may elaborate on their concerns in general as well as on hierarchical differences among these concerns. Secondly, while focusing exclusively hierarchical differences due to our research objective, there are further differences in stakeholder concerns within the same hierarchical level, e.g. CEO versus CIO, too. However, all interviewees were part of the IT side. Therefore, interviewees of a certain hierarchy level were relatively homogenous in our study. For a study comprising interviewees from the IT side as well as the business side, this is not imperatively the case. Finally, one limitation refers to the analysis of a single case study. There are still more concerns included in TOGAF, such as further quality criteria or architecture patterns, which are have not been reflected in the case at hand. For future research, more detailed insights from multiple stakeholder groups will become necessary to strengthen our findings and amplify the number of considered quality criteria.

Our findings led to several implications for future research. These concern potential improvements of EAM approaches, as it has been reflected on the example of TOGAF: The described three different granularity levels of architectures may be sufficiently covered by two, while some stakeholders were not interested in the granularity level at all. The quality term should be applied not only to architectural principles, but also to all deliverables. Moreover, different quality concerns need further refinement. Lastly, investigation for EAM advertising strategies will become a necessary focus for future research.

\section{REFERENCES}

[1] W. F. Boh and D. Yellin, "Using enterprise architecture standards in managing information technology," Journal of Management Information Systems, vol. 23, no. 3, pp. 163-207, 2006.

[2] Systems and software engineering -- Architecture description, ISO/IEC/IEEE 42010:2011, 2011.

[3] The Open Group, TOGAF Version 9.1, 1st ed. Zaltbommel: Van Haren Publishing, 2011.

[4] S. Kurpjuweit and R. Winter, R, "Concern-oriented business architecture engineering," in Shin, D. (Eds.): Applied Computing 2009 - The 24th
Annual ACM Symposium on Applied Computing, Honolulu, Hawaii, USA, 08.03.2009, ACM, 2009, pp. 265-272..

[5] Å. Lindström, P. Johnson, E. Johansson, M. Ekstedt, and M. Simonsson, "A survey on CIO concerns: Do enterprise architecture frameworks support them?," Information Systems Frontiers, vol. 8, no. 2, pp. 81-90, 2006.

[6] H. Jonkers et al., "Concepts for modelling enterprise architectures," International Journal of Cooperative Information Systems, vol. 13, no. 3, pp. 257-287, 2004.

[7] S. A. Bernard, An Introduction to Enterprise Architecture, 2nd ed. Bloomington, IN: AuthorHouse, 2005.

[8] R. E. Freeman, Strategic Management: A Stakeholder Approach. Cambridge: Cambridge University Press, 1984.

[9] R. E. Freeman, J. S. Harrison, A. C. Wicks, B. Parmar, and S. de Colle, Stakeholder Theory: The State of Art. Cambridge: Cambridge University Press, 2010.

[10] T. Donaldson and L. E. Preston, "The stakeholder theory of the corporation: Concepts, evidence, and implications," Academy of management Review, vol. 20, no. 1, pp. 65-91, 1995.

[11] T. J. Rowley, "Moving beyond dyadic ties: A network theory of stakeholder influences," Academy of management Review, vol. 22, no. 4, pp. 887-910, 1997.

[12] K. Buysse and A. Verbeke, "Proactive environmental strategies: A stakeholder management perspective," Strategic Management Journal, vol. 24, no. 5, pp. 453-470, 2003.

[13] K. Haki, S. Aier, and R. Winter, "A stakeholder perspective to study enterprise-wide IS initiatives," in 24th European Conference on Information Systems (ECIS), 2016.

[14] B. van der Raadt and H. van Vliet, "Designing the enterprise architecture function," in Lecture Notes in Computer Science, Quality of Software Architectures. Models and Architectures, S. Becker, F. Plasil, and R. Reussner, Eds.: Springer, 2008, pp. 103-118.

[15] S. Buckl, A. M. Ernst, F. Matthes, R. Ramacher, and C. M. Schweda, "Using enterprise architecture management patterns to complement TOGAF," in Enterprise Distributed Object Computing Conference: IEEE, 2009, pp. 34-41.

[16] P. Aleatrati Khosroshahi, M. Hauder, A. W. Schneider, and F. Matthes, Enterprise Architecture Management Pattern Catalog, 2nd ed., Software Engineering for Business Information Systems, 2015.

[17] M. M. Lankhorst, Enterprise Architecture at Work: Modelling, Communication and Analysis. Berlin: Springer, 2005.

[18] R. K. Yin, Case Study Research: Design and Methods, 5th ed. Thousand Oaks, London, New Delhi: Sage Publications, 2013.

[19] M. Q. Patton, Qualitative Research \& Evaluation Methods, 3rd ed. Thousand Oaks: Sage Publications, 2002.

[20] M. B. Miles and A. M. Huberman, Qualitative data analysis: An expanded sourcebook. Thousand Oaks: Sage Publications, 1994.

[21] K. M. Eisenhardt, "Building theories from case study research," Academy of management Review, vol. 14, no. 4, pp. 532-550, 1989.

[22] A. K. Schnackenberg and E. C. Tomlinson, "Organizational transparency: A new perspective in managing trust in organizationstakeholder relationships," Journal of Management, vol. 42, no. 7, pp. 1784-1810, 2014. 